Research Paper

\title{
Two Olfactory Pathways to Detect Aldehydes on Locust Mouthpart
}

\author{
Liwei Zhang, Hongwei Li and Long Zhang ${ }^{\bowtie}$ \\ Department of Entomology, China Agricultural University, Beijing 100193, PR China \\ $\triangle$ Corresponding author: locust@cau.edu.cn; Tel: +86-10-62731303; Fax: +86-10-62731048 \\ (c) Ivyspring International Publisher. This is an open access article distributed under the terms of the Creative Commons Attribution (CC BY-NC) license \\ (https://creativecommons.org/licenses/by-nc/4.0/). See http://ivyspring.com/terms for full terms and conditions.
}

Received: 2017.02.26; Accepted: 2017.03.29; Published: 2017.05.27

\begin{abstract}
Sensing chemical cues is crucial for insects through their olfactory systems to adapt the environments. The receptors employed in insect olfactory system belong to the Odorant Receptor (ORs) and lonotropic Receptor (IRs) families. In general, ORs and IRs are present in distinct olfactory sensory neurons and function independently. Here, we present evidence that in locust, the abundant host plant odor Hexanal is detected by both IR- and OR-expressing neurons. Use of the palp opening response (POR) as a simple behavioral paradigm in conjunction with RNA interference (RNAi) revealed that these two pathways are both needed for the detection of Hexanal. Two-color fluorescence in situ hybridization showed that OR2 and odorant-binding protein 1 (obpl) were co-localized in palps sensilla basiconica. Obp2a and IR8a were co-localized as well, but associated with sensilla chaetica on the palps. Furthermore, both OR2- and obpl-knockdowns showed reduced POR responses to Hexanal and E-2-Hexenal, and the same was true for Hexanal with IR8a- and obp2a-knockdowns. Detection to E-2-Hexenal was independent of IR8a-mediated gene silencing. Besides, Hexanal and E-2-Hexenal evoked dose-dependent responses in palp basiconica via extracellular recordings. Our results indicate that both OR and IR pathways are involved in the detection of one aldehyde.
\end{abstract}

Key words: palp opening response, odorant receptor, inotropic receptor, odorant-binding protein, RNAi, aldehydes.

\section{Introduction}

Olfaction plays an essential role in insects' survival and reproductive success, and also mediates responses to food, enemies and mates. The two most important olfactory appendages, antenna and palps are covered with sensory hairs called sensilla, which house olfactory receptor neurons (ORNs). On the ORNs dendritic membrane, odorant receptors (ORs) and ionotropic receptors (IRs) detect and discriminate distinct odorants and further induce neuronal depolarization and ion flux across the cellular channels [1-4]. Insect ORs are a recently evolved chemosensory family that exhibits inverse topological structure compared to mammalian homologs $[5,6]$. A functional OR complex consists of multimers of two subunits, the universal co-receptor, Orco and a tuning OR. The neuronal depolarization mechanism of
OR-expressing cells remains controversial, and at least two theories are available regarding ionotropic and metabotropic signaling pathways [7, 8]. In addition, antennal ionotropic receptors have been characterized as an ancient olfactory receptor family originating from ionotropic glutamate receptors (iGluRs) [9], and IRs tune mostly to acids and amines [10]. Even though both IRs and ORs are expressed abundantly on olfactory organs, few reports showed these ORNs are co-localized in same sensilla except for Drosophila antennal coeloconica type 3. In the ac3B neurons, one OR complex (OR35a and Orco) is co-expressed with IR76b $[3,11]$. In addition, mosquito vector Anopheles gambiae larvae rely on two distinct olfactory signaling pathways for chemotaxis movements: one system depends directly on ORs, 
while the other is OR-independent and requires the existence of IRs [12]. This highly compartmentalized distribution pattern indicates functional differentiation with potential behavioral significance.

Locust is a model organism among hemimetabolous insects, which are different from the holometabolous Drosophila. The orthopteran locust represents a key link in molecular evolution of olfaction [13]. Previous observations suggested that locust palps play an important role in food selection, especially for the detection of non-volatile chemical cues [14-18]. Additionally, on the dome of the distal segment of palps, olfactory basiconica are identified [19] and one odorant receptor, OR2 appears to be expressed in a small set of sensory neurons on the maxillary palps [20]. Besides, a set of various odorant binding proteins were identified on the palps as well $[19,21,22]$. All of these observations indicate that palps seem to receive olfactory cues, probably related to food, and integrate signals with antennal parallels [23]. However, there is no information on the expression pattern of receptors and also the chemical cues coded by this underestimated appendage. Though the genetic toolkit is largely limited in locust, RNA interference (RNAi) was demonstrated to be reliable in systematical silencing [24]. In this study, we demonstrated a new, efficient behavioral paradigm for the assessment of palp olfactory detection, and proved the involvement of OR and IR in conjunction with RNAi. Our results provide a new view on interaction between ORs and IRs for detection of one odorant, with implications on the evolution of locust olfaction system in contrast to fly and moth.

\section{Material and methods}

\section{Locust}

Migratory locusts, Locusta migratoria menilensis (Orthoptera) were raised under crowded condition in the Department of Entomology, China Agricultural University with relative humidity of $60 \%$ and temperature of $28-30^{\circ} \mathrm{C}$ under photoperiod of $18: 6 \mathrm{~h}$ (light : dark). Locusts were fed with fresh wheat seedlings every day. For RNA isolation, target tissues were isolated and immediately stored into liquid nitrogen or kept under $-80^{\circ} \mathrm{C}$ for long term storage.

\section{Chemicals and preparation}

All chemicals used for behaviors were ordered with highest purity. Working solutions were prepared with paraffin oil. All chemical compounds in this research were listed in Table S2.

\section{Classical olfactometer assay}

Sufficient fresh wheat leaves were ground and then the supernatant was collected after centrifugation. The crude extract was prepared by $1 / 10$ dilutions with dichloromethane into the supernatant. Filter paper ( $3 \mathrm{~cm}$ wide, $30 \mathrm{~cm}$ long) was loaded with $100 \mu \mathrm{l}$ extracts as treatment or same amount of solvent as control to each side at $7 \mathrm{~cm}$ distant from the entrance hole on the bottom of the arena. Filter paper loaded with chemical or solvent were replaced with new one after previous test. 5th-instar animals were individually introduced into the arena. The activity of locust was recorded by an overhead camera for 5 minutes at $28-30^{\circ} \mathrm{C}$ under illumination. Selection preference was defined that once locusts firstly moved toward one side and stayed over 15 seconds. Recordings from locusts without unambiguous selection in 5 mins were discarded. The video was analyzed with Ethovision XT 8.0 (Noldus, Netherlands).

\section{Palp opening response (POR)}

4-6 days old $5^{\text {th }}$ instar nymphs were used for POR assay. In brief, starved animal (10 hours) with antennae ablation was restrained within truncated 1.5 $\mathrm{mL}$ Eppendorf tubes and its head and palps could move freely. All tubes were immobilized on a rack to facilitate fast testing toward animals in the warm hood $\left(28-30^{\circ} \mathrm{C}\right)$. Before POR, all animals were warmed at least 10 minutes for adaptation. Each experimental group contains 30 locusts, and $2 \times 10 \mu$ l working solution was applied onto filter paper strip $(2 \mathrm{~cm} \times 0.5$ $\mathrm{cm}, \mathrm{L} \times \mathrm{W})$. A fast approaching was made toward locust mouthparts but without contacting palps and it's observed if one or more palps extended over labrum sulcus during stimulation. Locust number opening palps was counted. Each experimental group was replicated three times with an interval of 15 minutes to recover palp activity and all datasets were pooled and averaged to minimize variations. POR assays of gene regulation groups were replicated at least with three independent groups. POR index was defined as total tested insects divided by number of responsive individuals.

\section{Semi-quantitative RT-PCR}

Semi-quantitative RT-PCR was used to check the silencing efficiency from gene-specific RNA interference. Briefly, $1 \mu \mathrm{g}$ of total RNAs from various RNAi treatments and control tissues were transcribed into cDNA (FastQuant RT Kit, TianGen, China). For each independent PCR, gene specific primer pairs were designed (see Table. S1) and equivalent amount of cDNA was used as template for amplification (TaKaRa Ex Taq, TaKaRa, Japan). Two-step PCR was run with the program including pre-denature for 30 sec at $95^{\circ} \mathrm{C}$ followed with 40 cycles $\left(95^{\circ} \mathrm{C}\right.$ for $5 \mathrm{sec}$ and $60^{\circ} \mathrm{C}$ for $30 \mathrm{~s}$ ). 
In order to relatively compare expression level, $20 \mu \mathrm{l}$ of PCR product was imaged on $1.5 \%$ agarose gel. All primers designed for semi-quantitative RT-PCR are listed in Table S1.

\section{In situ hybridization}

Single and two-color in situ hybridization was performed on tissue cryosections (12-14 $\mu \mathrm{m})$. Standard protocols for in situ hybridization were modified partially [20, 29, 31]. Briefly, probes for each gene were prepared by gene amplification (Primer sequences see Table. S1) and cloning into pGEM-T /-T easy vector for subsequent linearization. For single color hybridization, digoxigenin (Dig)-labeled antisense probes in $100 \mu \mathrm{l}$ hybridization solution were used to treat tissue sections overnight, and then anti-digoxigenin alkaline phosphatase (AP-) conjugated anti-body (Roche, diluted 1:500 in 1\% blocking buffer) was added on sections. Subsequently, hybridization signals were visualized using NBT (Nitroblue tetrazolium) and BCIP (5-brom-4-chlor-3-indolyl phosphate). Tissue sections were analyzed on Olympus IX71 microscope (Olympus, Tokyo, Japan). Two-color hybridization was conducted similar to single color staining. Dig-labeled antisense probes (obp1 and obp2a, 1:300 dilutions) were visualized by the anti-Dig AP-conjugated antibody (1:500 dilutions) in combination with HNPP/Fast Red (Roche). For biotin-labeled antisense probes (Orco, OR2 and IR8a, 1:100 dilutions), the TSA kit (Perkin Elmer, MA, USA), including an anti-biotin strepavidin horse radish peroxidase-conjugate and fluorescein-tyramides as substrate (1:100 dilutions) was used. Images were captured on Olympus BX45 confocal microscope (Olympus, Tokyo, Japan) and analyzed using FV1000 software.

\section{RNA interference (RNAi)}

Double stranded RNA (dsRNA) was synthesized based on manufacturer manual. In brief, PCR products (between 300-700 bp, primer pairs see Table. S1) were amplified with T7 promoter conjugated primer, and then purified with Wizard ${ }^{\circledR}$ SV Gel and PCR Clean-Up System (Promega, USA) as templates for in vitro transcription. DsRNA was synthesized with T7 RiboMAX ${ }^{\mathrm{TM}}$ Express RNAi System (Promega, USA) and diluted into $1000 \mathrm{ng} / \mu \mathrm{l}$ with ddH2O and stored at $-20^{\circ} \mathrm{C}$. Target dsRNA $(5 \mu \mathrm{g})$ and control $(5 \mu \mathrm{l}$ ddH2O) was delivered into each locust dorsal vessel through inter-segmental membrane (first day of $5^{\text {th }}$ instar nymph) by IM-9B microinjector (Narishige, Japan) equipped with glass capillary. For double injection assays, dsRNA of each gene was diluted into $2000 \mathrm{ng} / \mu \mathrm{l}$ first and then mixed completely before injection. DsGFP was microinjected as control group. The treated locusts were raised normally like wild type animals. RNA silencing was checked between $3^{\text {th }}$ and $6^{\text {th }}$ day post-injection. All RNAi-treated animals used for behaviors were checked after POR to confirm the silencing.

\section{Extracellular recording}

Single Sensillum Recordings (SSRs) and chemical stimulations were performed on the maxillary palp of $5^{\text {th }}$ instar female nymph. The locust was amounted with tape and plasticine on the glass slide and one of its maxillary palps was immobilized with thin tungsten on a piece of cover slide. Tungsten electrodes were sharpened electrolytically with $10 \%$ $\mathrm{NaNO} 2$ under the microscope. The recording electrode was inserted in the base of basiconic sensillum by using a motorized micromanipulator (CFT-8301D, C.M.D.T, China) and an indifferent electrode was inserted into the head. All stimulation duration is $1 \mathrm{sec}$. The recording electrode was connected to a 10X universal AC/DC amplifier (Syntech, The Netherlands). The recording signals were collected on Intelligent Data Acquisition Controller (IDAC-4, Syntech, the Netherlands) and were viewed on a personal computer. Software AUTOSPIKE (Syntech, the Netherlands) was used to quantify the extracellular responses recorded for either $1 \mathrm{sec}$ or $5 \mathrm{sec}$, before and after each stimulation. All statistical analysis was performed with GraphPad Prism 7 (Graphpad software, San Diego, California, USA).

\section{Statistical analysis}

Data from classical olfactometer were analyzed by exact binomial tests and two-way ANOVA with Bonferroni post-tests were used for POR assays. Extracellular recordings were quantified with one-way ANOVA with post hoc $t$ tests. All data were analyzed using GraphPad Prism 7 (Graphpad software, San Diego, California, USA).

\section{Results}

\section{Mouthparts are not involved in long-distance orientation}

The first goal of this study was to establish reliable behavioral paradigms that were needed to quantify behavioral outputs after odorant stimulation. Previously, use of a classical olfactometer for this purpose was reported in phase-related behavior analysis in locust [25]. We updated the setup by adding a camera to track locusts in the experimental arena. Via this way, movement of insects to either chemical- or solvent-loaded areas was quantified. To investigate whether olfactory inputs via antennae and 
palps contribute to behavior, either or both were ablated. As shown in Figure 1A, intact locusts were attracted significantly more by wheat volatiles than solvent, which means locusts were able to distinguish food odors. When palps were ablated but antennae kept intact (Figure 1B), animals behaved similar to those with both tissues intact, and were attracted to the food source. This likely argues that palps olfactory input did not substantially contribute to the attractiveness to food odors. When antenna were removed instead of palps (Figure 1C), locusts did not choose preferentially between food odors and solvent. This implies that the antennal olfactory system is necessary for functional distinction of food cues. It needs to be noted that previous reports stated that locusts respond with palpation (palp extending and vibrating) to stimulus when situated in very close range; the comparatively long distance in the setup might have caused the apparent lack of palp involvement [26]. To address this, we shortened the distance to the odor source in the setup. However, we found that locusts with antennal ablation responded with emesis to airborne stimulation of E-2-Hexenal under high concentration $(50 \% \mathrm{v} / \mathrm{v}$, in paraffin oil, Video S1) at close proximity; the solvent didn't cause this acute reaction (Figure 1D, E, Video S2). This indicated that the palps possibly respond to volatile compounds under high stimulation intensity over short distance.

\section{Quantification of olfactory responses of mouthparts through palp opening response (POR)}

Then we asked whether palps are able to detect odors at low concentrations. Antennae-ablated animals were tested at $5 \%(\mathrm{v} / \mathrm{v})$ dilutions. For analysis, we defined a palp opening response (POR) when animals extend any appendage from two palp pairs beyond the prolonged cracks (Figure 2A, dashed lines) connecting sutures [27]. One positive POR example was provided when locust was treated with the corn leaf volatile, 2-Heptanone $(5 \% \mathrm{v} / \mathrm{v})$. This odor excited locust to extend palps without emesis (Figure 2B, C, Video S3), while palps were unresponsive toward solvent (Figure 2B, D, Video S4). Similarly, another leaf volatile, Z-3-Hexenol elicited a significantly higher POR percentage over solvent (Figure 2C). Z-3-Hexenyl acetate (HA) is sensed by antenna with high sensitivity based on electroantennogram responses [28]. However, similar to paraffin oil, 5\% HA was unable to stimulate palps extension (Figure 2D). In summary, our POR bioassay indicated that certain green leaf volatiles (GLVs) are detected by palps under proper situations.

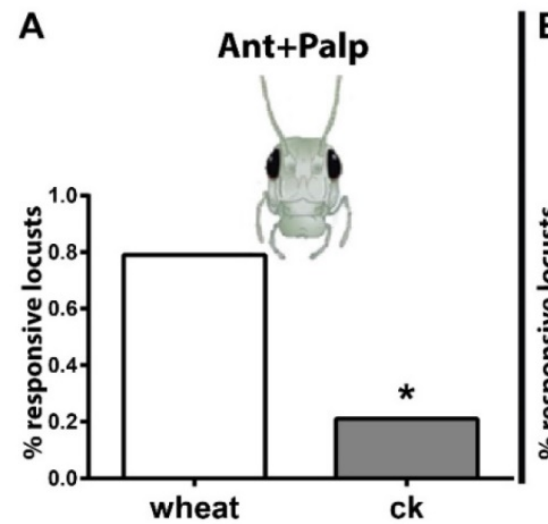

D

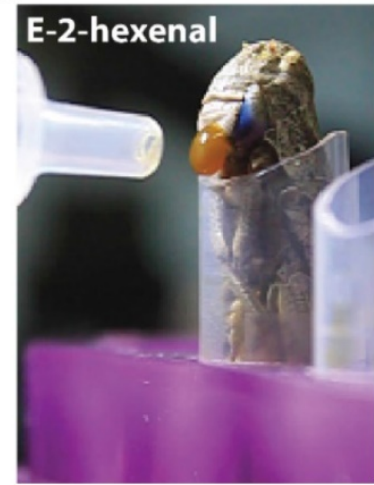

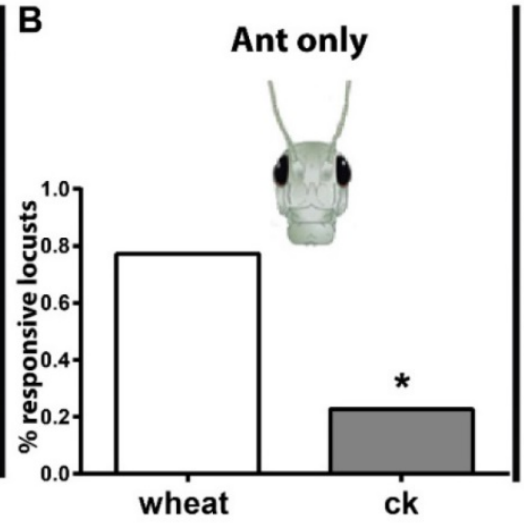
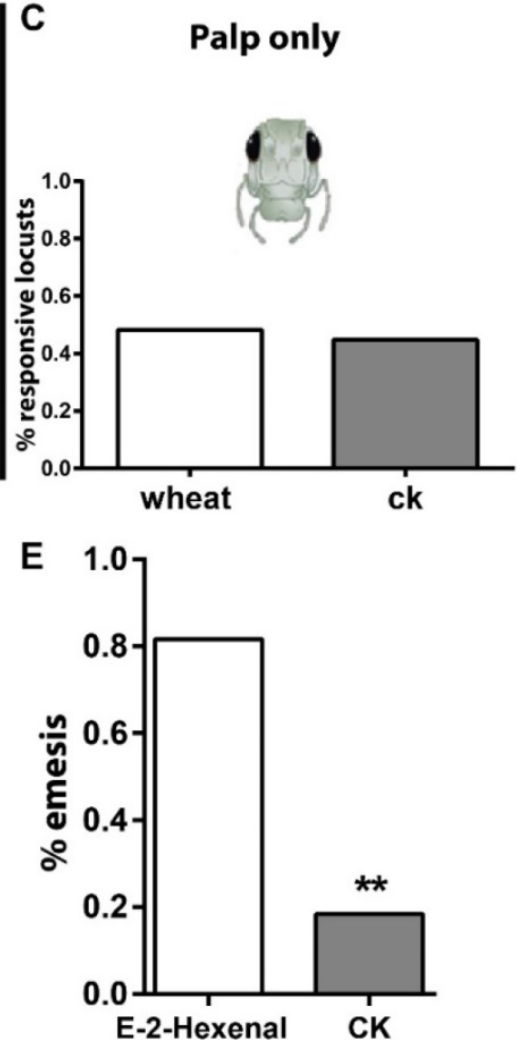

Figure 1. Locust antenna and mouthparts act different roles in olfaction. A-C Long-range behavioral choice toward wheat volatiles and CK vehicle (dichloromethane). $n=20,22$ and 29 locusts tested for $\mathbf{A}, \mathbf{B}$ and $\mathbf{C}$. Locust head cartoon modified from TutorVista.com. D schematic illustration for locust emesis challenged with $50 \% \mathrm{v} / \mathrm{v}$ E-2-hexenal and paraffin oil. E Emesis ratio of tested animals toward E-2-hexenal. $n=30$ locusts. * $\mathrm{p}<0.05$, $* * \mathrm{p}<0.001$, exact binomial tests 
A
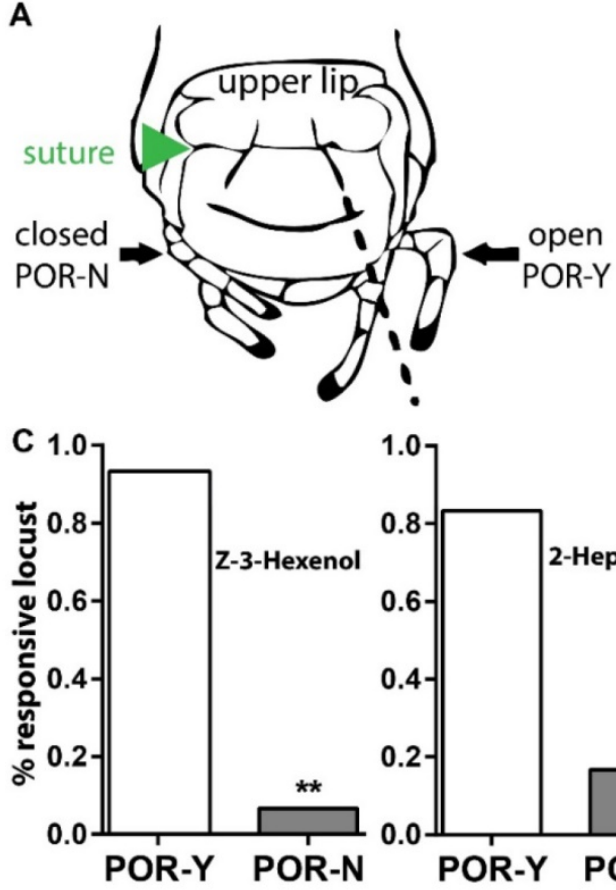
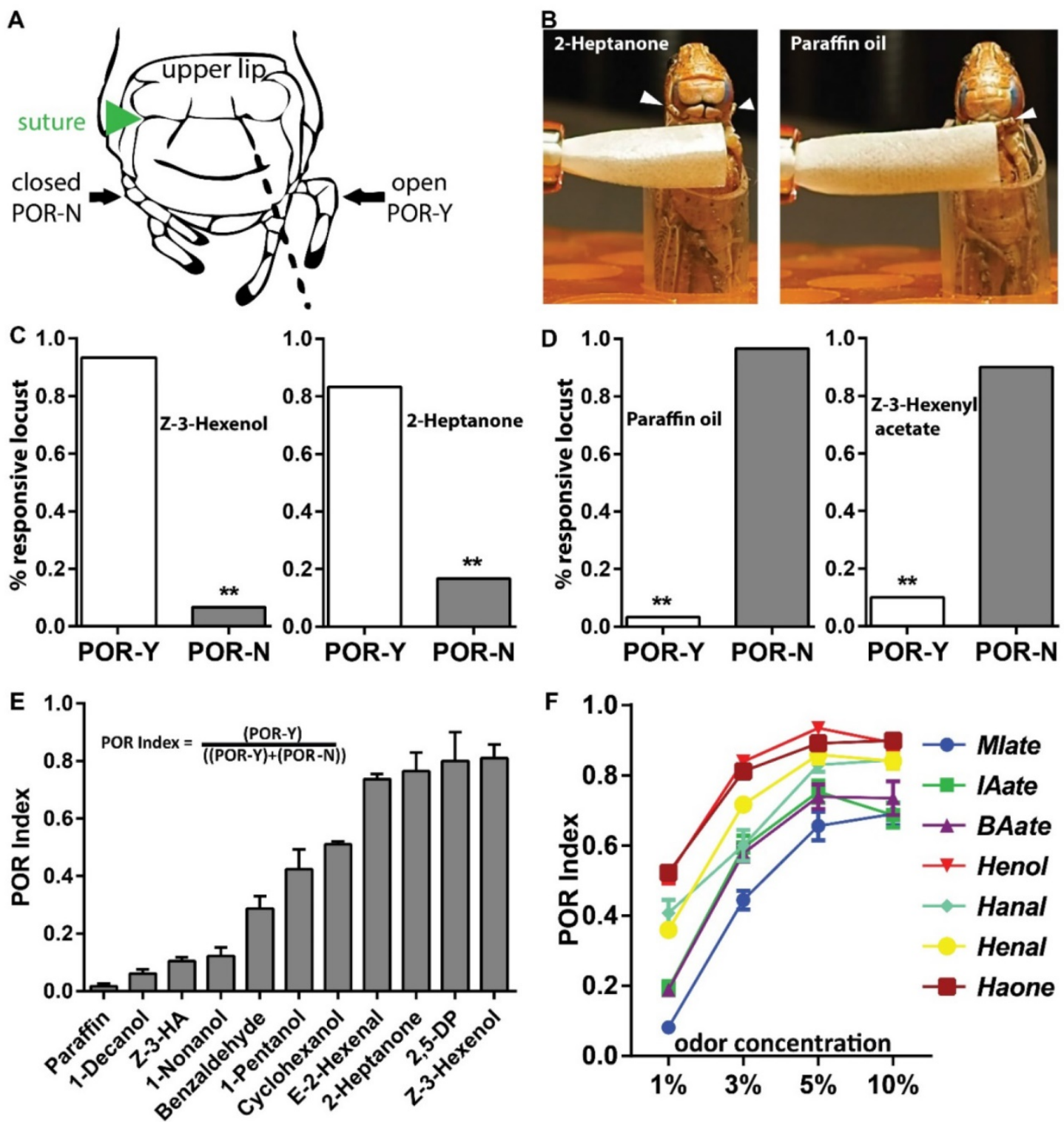

G

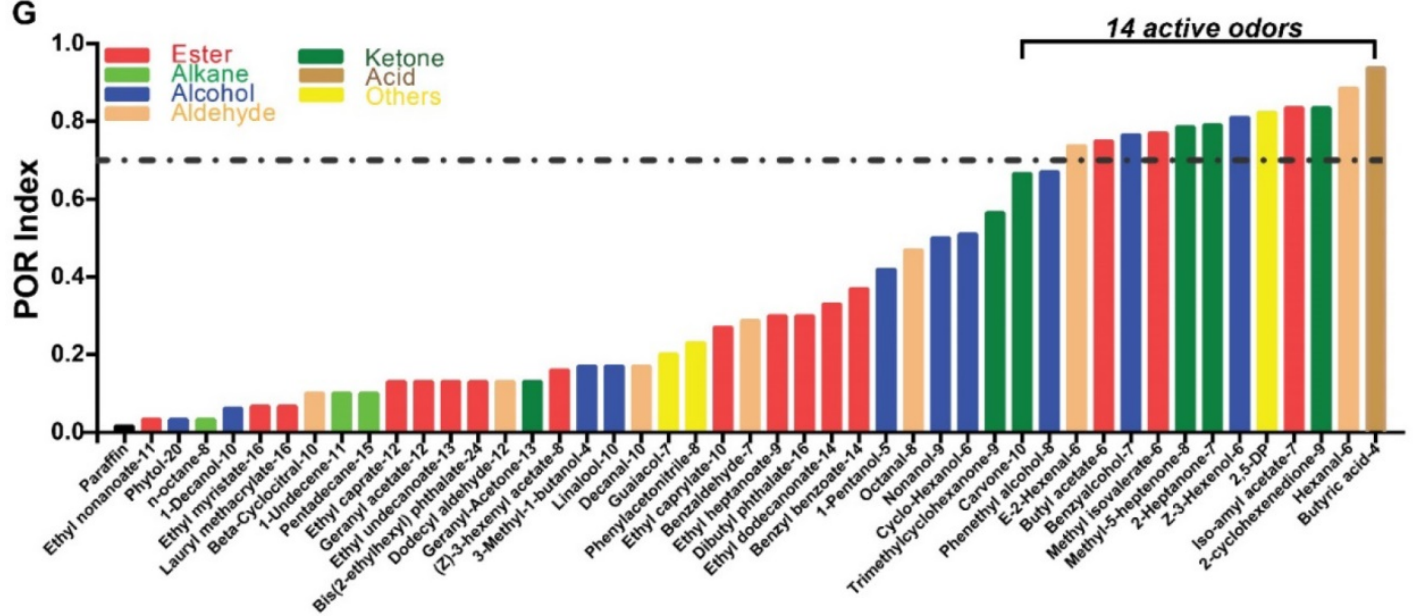

Figure 2. Palp opening responses (POR) quantify olfactory reactions on mouthpart. A Locust head structure illustrating plap's position during opening (POR-Y) and closing (POR-N). Locust head cartoon modified with permission from Simoes, et al [27]. B Schematic for POR when locust are tested with $5 \%$ (v/v) 2-Heptanone and paraffin oil. Arrowhead indicates palp position and filter paper loaded with compounds is in close proximity but never touch palp. C-D POR examples for active in $\mathbf{C}$ and non-active compounds in $\mathbf{D}$ at $5 \%$ ( $/ \mathrm{v}$ ) dilutions. $n>=30$ locusts tested in each assay. ${ }^{* *} \mathrm{p}<0.001$, exact binomial tests. E POR quantification of multiple compounds $(5 \%, \mathrm{v} / \mathrm{v})$ based on POR index (responsive locust number divided by total tested locust number). $\mathrm{n}=4$ ( 30 locusts each replicate) for each compound. Z-3-HA: Z-3-Hexenyl acetate; 2,5-DP: 2,5-Dimethylpyrazine. $F$ Dosage-curve of POR assays from 7 chemicals. $n=6-9$ (30 locusts each replicate) for each independent test. All error bars in E and F indicate S.E.M. Mlate: Methyl isovalerate; IAate: Iso-amyl acetate; BAate: Butyl acetate; Henol: Z-3-Hexenol; Hanal: Hexanal; Henal: E-2-Hexanal; Haone: 2-Hexanone. G Compound screens based on POR index. All compounds are tested at $5 \%$ (v/v) dilution in paraffin oil. $n=30$ locusts for each compound. Dashed line indicate POR index of 0.7 , criterial for active chemicals. All chemicals are end-flanked with carbon numbers and detailed information in Table. S2. 
Next we performed additional experiments with the goal of quantifying the response. Accordingly, POR-index was defined as the percentage of individuals displaying POR and noted difference toward additional odors (Figure 2E). Decanol, HA and Nonanol elicited weak POR, with a POR-index lower than 0.2. In contrast, Z-3-Hexenol showed strong index higher than 0.8. Furthermore, we examined the sensitivity and specificity of this index using 7 compounds containing various functional groups (including aliphatic esters, alcohols, aldehydes and ketones). As shown in Figure 2F, all tested compounds elicited enhanced POR intensity when concentrations were increased from $1 \%$ to $5 \%$, but with a weak decrease at $10 \%$. This indicates palps distinct volatile information in a dose-dependent manner. On the other hand, Z-3-Hexenol and 2-Heptanone elicited the maximal POR index across all test concentrations; while aliphatic esters, including Iso-amyl acetate, Methyl isovalerate and Butyl acetate failed to elicit as strong responses as the additional compounds in the compound set. Next, we enlarged chemical set including sources of host plants, locust cuticular and fecal volatiles and checked their bio-activity in POR (Figure 2G). Interestingly, locust palps exhibited the ability to recognize and distinguish a number of chemicals. In total, we identified 14 active compounds with a POR index higher than 0.7. Remarkably, all these compounds have a limited number of carbon atoms (6 to 9) and they fall into several chemical groups, such as aliphatic esters, alcohols, aldehydes, ketones and heterocyclic compounds. Taken together, this simple bioassay provides a way to quantify olfactory responses on mouthparts sensitively and specifically, without interference from antennae.

\section{Identification of OR2 and obp 1 required for detection of two aldehydes}

Peripheral odorant detection involves OBPs, ORs and IRs in the olfactory sensilla. Since several olfactory proteins were identified on locust palp [19, $20,29,30]$, we employed in situ hybridization to characterize their cellular localization. In single color staining, antisense probes against Orco labeled sparse cell clusters (Figure S1B); each cluster contained multiple sensory neurons in a pattern similar to antennal basiconica [31]. On the palp dome, only 5-7 porous basiconica are found, and it seems plausible that Orco is exclusively expressed in basiconica ORNs. Additionally, we checked obp1, the first identified OBP in locust and its probes labeled cavity-like structures and no neurons were labeled (Figure S1A1-3). Furthermore, signals in two-color hybridization marking Orco and obp1 did not overlap fully, but were in close proximity to each other (Figure $3 \mathrm{~A}$, top). Since OBPs are secreted from support cells surrounding ORNs; obp1 and Orco are likely expressed in neighboring cells underneath same palp basiconica. $\mathrm{Xu}$, et al reported sparse distribution of OR2-expressing cells on the maxillary palps [20] and that possibly represents functional localization in basiconica neurons. Two-color hybridizations marked positive cells expressing either OR2 or obp1 respectively (Figure 3A, bottom). One OR2-expressing cell was enclosed inside the pocket structure labeled by obp1; this distribution was restricted to baciconica instead of the abundant chaetica. These observations indicate that OR2 and obp1 are associated in the same palp basiconica, together with Orco.

To further check if obp1/OR2 complexes contribute to olfaction functionally, animals were treated with dsRNA against either gene to specifically down regulate expression. Wild type insects exhibit higher gene expression on antennae in comparison to palps, likely due to larger numbers of basiconica on antennae (Figure 3B). Once injected with dsRNA, transcripts in both organs were inhibited largely, indicating success of systematical interference. Subsequently, injected animals were examined with 7 active compounds previously screened (except where stated, otherwise $5 \% \mathrm{v} / \mathrm{v}$ was used in all assays). Compared with water-injection, treatment of dsobp1 led significantly attenuated POR-indices toward two aldehydes, Hexanal and E-2-Hexenal, but with residual responses (Figure 3C). The remaining compounds elicited normal behavior under suppression of obp1. This argues that lacking obp1 impaired palp olfactory responses specifically to these aldehydes. We further checked influences on POR after silencing ORs (Figure 3D, E). Similarly, locusts with either receptor impairments exhibited significantly reduced activity to aldehydes. Silencing Orco did not affect POR for any compounds except for aldehydes; it seems likely that either Orco-independent pathways transduce chemical signals, or that the residual Orco protein is sufficient for normal reaction under experimental conditions. DsRNA against GFP was used as independent control and no significant index changes to any tested compound were observed in comparison to untreated insects (Figure 3F). This means the reduced POR to aldehydes was due to molecular regulations of obp1, OR2 and Orco. Finally, RT-PCR results ruled out the possibility of artifacts by cross-interference between these three genes in RNAi (Figure 3G). We conclude that OBP1, OR2 and Orco are involved in olfactory detection of aldehydes by the mouthparts. 
A
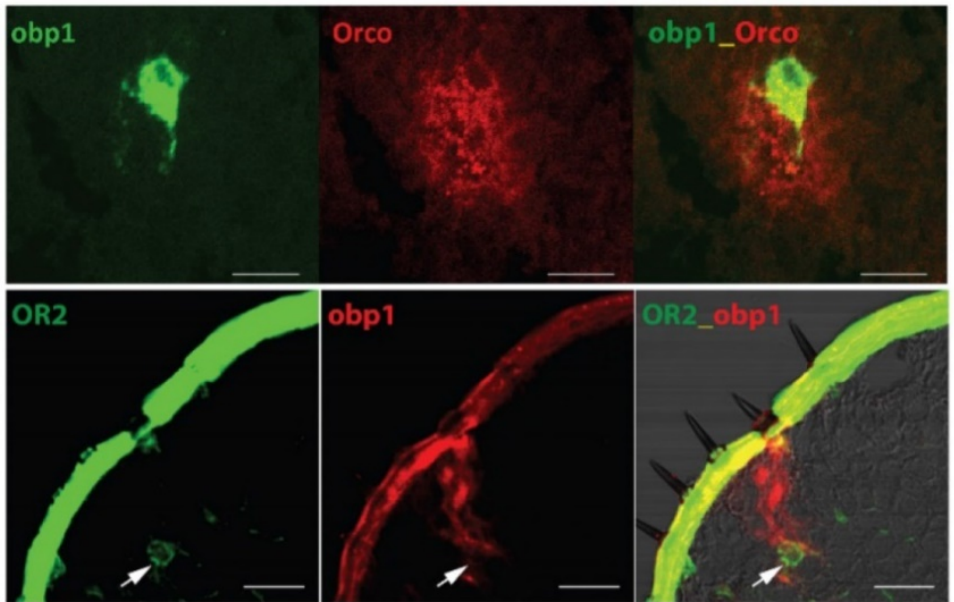

C

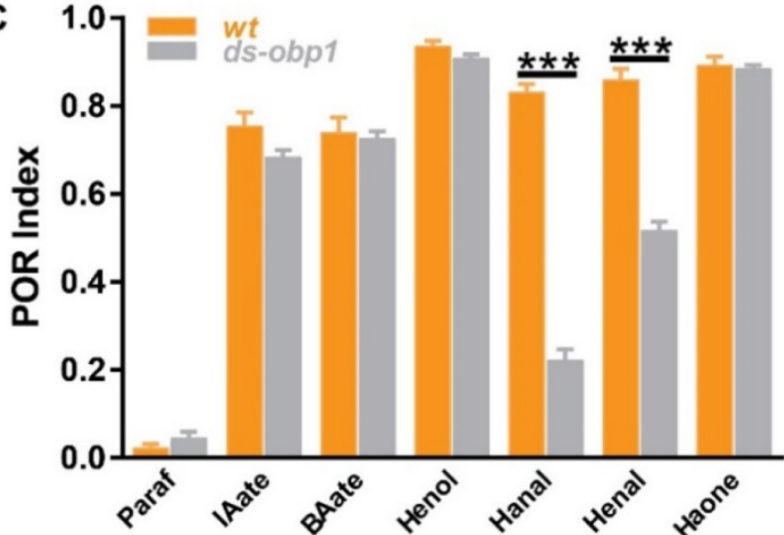

E

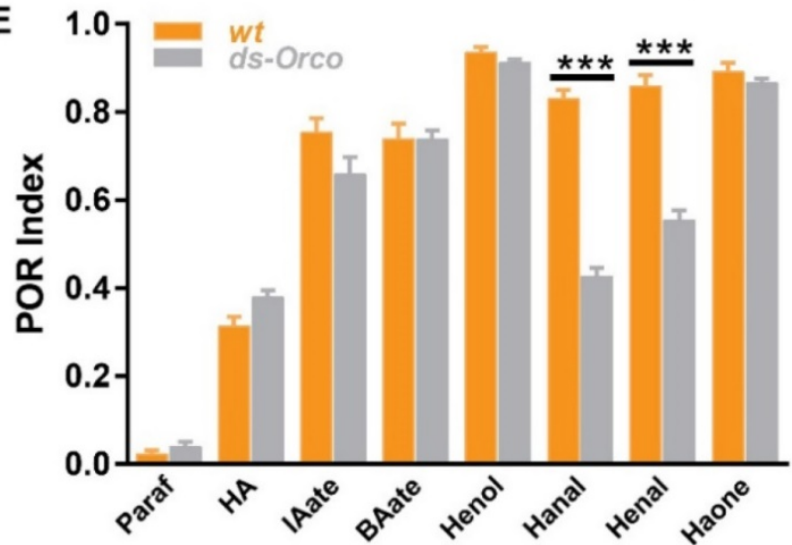

G

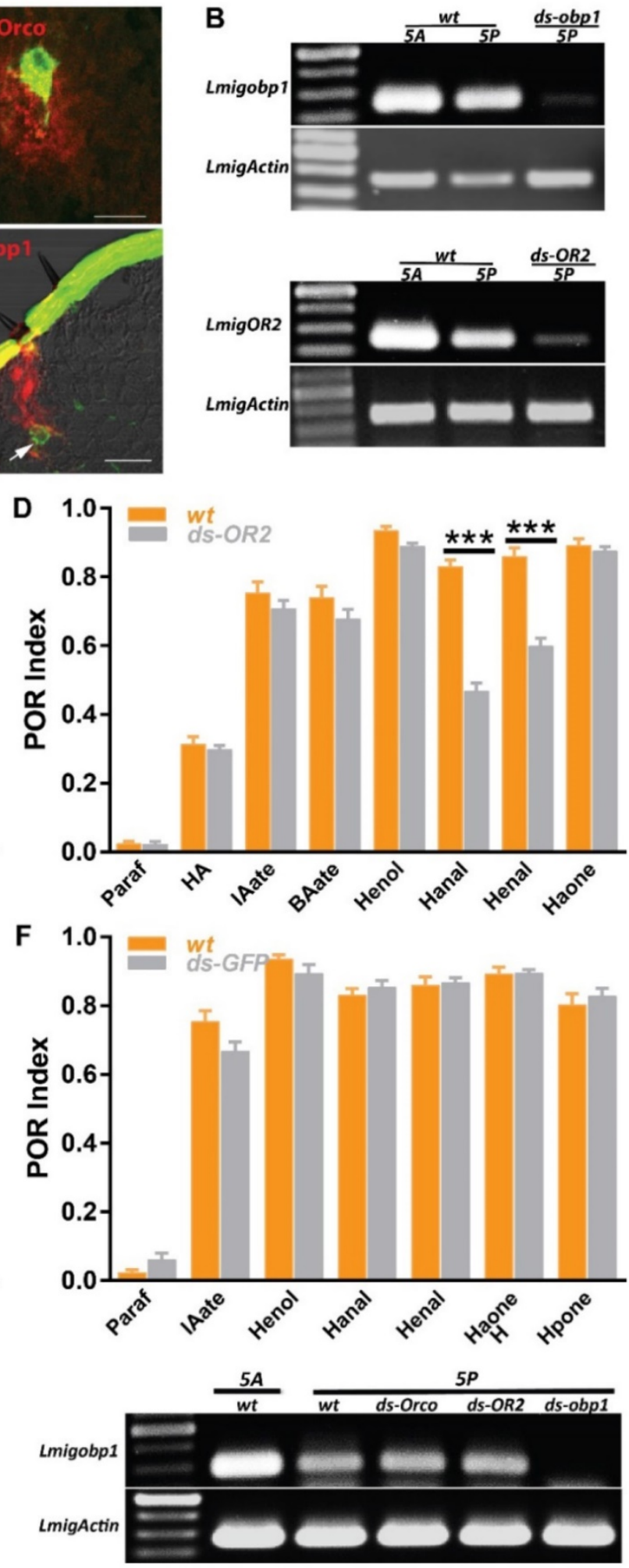

Figure 3. Odorant receptor pathways mediate two aldehyde detections on the mouthparts. A Two-color fluorescence in situ hybridization on palp and antennae cryosections. Top, co-expression of obpl and Orco in same palp sensilla; Bottom, co-expression of obpl and OR2 in same palp basiconica. White arrow indicates cell expressing OR2. Bar, $40 \mu \mathrm{m}$. B Semi-quantification RT-PCR to check the silencing efficiency of RNAi against obp1 and OR2 in 5 th instar nymph. wt, water injected; ds-obpl, dsRNA of obpl injected; ds-OR2, dsRNA of OR2 injected. 1 g total RNA used for RT in both samples. Actin was used to check template quality. C POR assays toward multiple compounds with locusts treated with water (wt) or ds RNA of obpl. $n=9$ (30 locusts each replicate) for each assay. D POR assays toward multiple compounds with locusts treated with water (wt) or ds RNA of OR2. $n=9-11$ (30 locusts each replicate) for each assay. E POR assays toward multiple compounds with locusts treated with water (wt) or ds RNA of Orco. $\mathrm{n}=9-12$ (30 locusts each replicate) for each assay. F POR assays toward multiple compounds with locusts treated with water (wt) or ds RNA of GFP. $n=5-9$ (30 locusts each replicate) for each assay. Error bar indicates S.E.M. *** $\mathrm{p}<0.001$, two-way ANOVA with Bonferroni post-tests. G No cross-silencing within Orco pathways with semi-quantitative RT-PCR. Actin was used to check template quality. Abbreviations are referred to B. Paraf: Paraffin oil; HA: Z-3-hexenyl acetate; IAate: iso-amyl acetate; BAate: Butyl acetate; Henol: Z-3-Hexenol; Hanal: Hexanal; Henal: E-2-Hexenal; Haone: 2-Hexanone; Hpone: 2-Heptanone 


\section{Extracellular responses in mouthparts sensilla basiconica tuned to two aldehydes}

To further testify these two aldehydes are truly detected via olfactory basiconica on the palp, extracellular responses of basiconica ORNs to E-2-Hexenal and Hexanal were recorded. At least two sensilla subtypes (we named them as pb1 and pb2) containing ORNs that were activated on the basis of diagnostic compounds (1-Nonanol and Nonanol acid), were identified and each of them showed differential response dynamics: neurons in pb1 produced significantly more spikes to 1-Nonanol than Nonanoic acid; however, pb2 neurons were significantly less activated by 1-Nonanol when compared to Nonanoic acid (Figure 4A, B). However, it is challenging to attribute the single compounds to each Olfactory Receptor Neurons (ORNs) inside pb1 and $\mathrm{pb} 2$ concerning that up to 15 sensory neurons were observed inside one palp basiconica[19]. That means that the spikes with similar amplitude could be from different ORNs and the larger spikes could be summed from multiple spontaneous activities of various ORNs. Besides, E-2-Hexenal evoked long-lasting excitements in pb1 neurons, rather than in pb2, which is much less activated during long recordings; however, both subtypes conferred similar robustness to Hexanal and it's challenging to tell them from each other functionally. Finally, dose-curve recordings were used to check response sensitivity evoked by two aldehydes in pb1 and pb2 and clear increments along with higher concentration were observed in all cases (Figure 4C, D). All of that indicated more than one coding patterns underlying specificity to aldehydes and these functional patterns derived from palp basiconica subtypes.

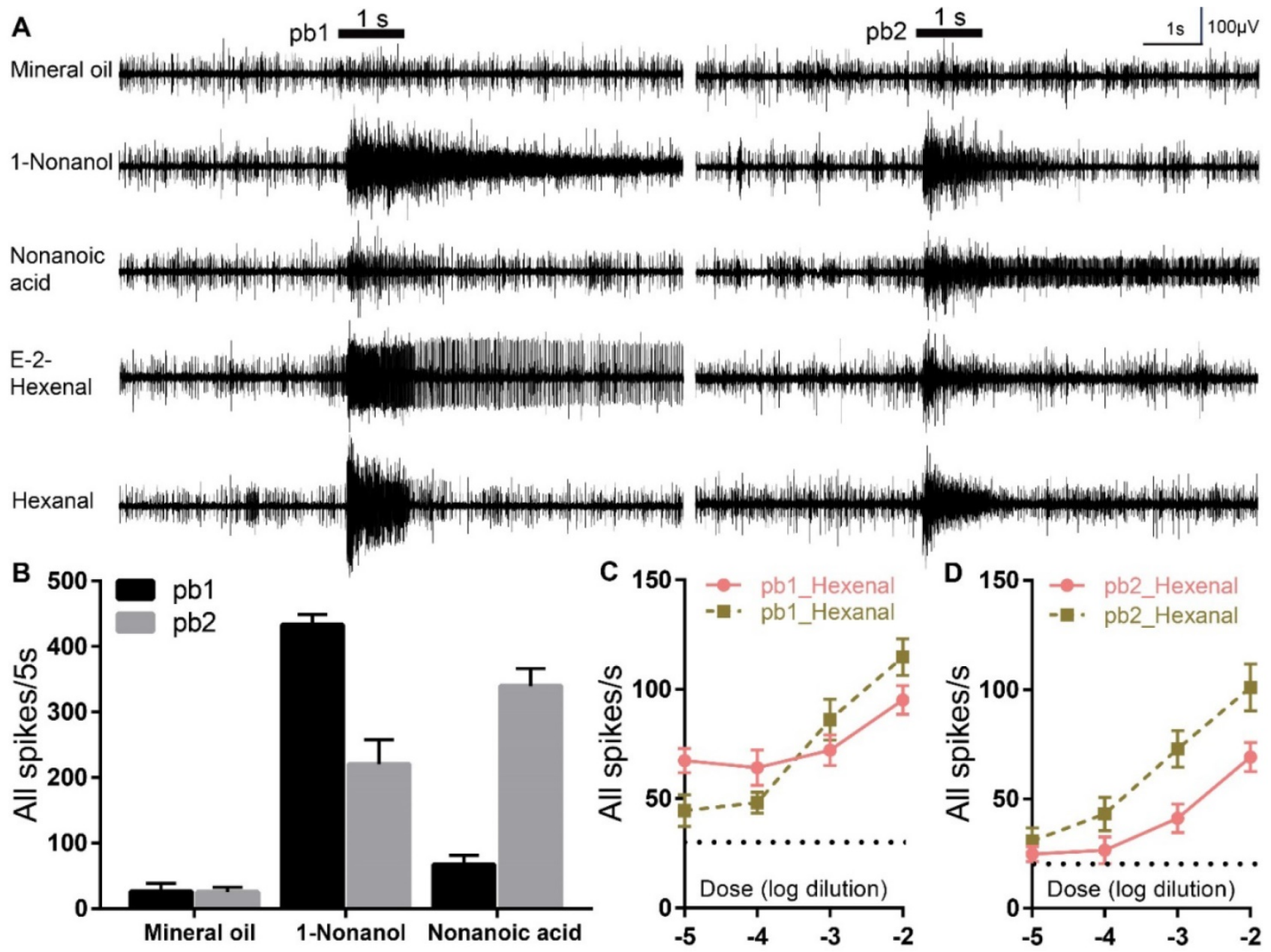

Figure 4. E-2-Hexenal and Hexanal elicit differential neuronal activities in palp basiconica. A Representative traces from extracellular recordings to the indicated stimuli in palp basiconica 1 (pbl) and palp basiconica 2 (pb2). Bars above the traces mark stimulus duration (1 s). For these recordings, all odors were used at $10 \%$ dilutions except for E-2-Hexenal and Hexanal, which were diluted at 1\%. B Quantification of mean changes of all spikes in $5 \mathrm{~s}$ before and after stimulus. In pbl, response to $1-$ Nonanol is significantly higher than Nonanoic acid ( $n=11$ palps; ANOVA with post hoc $t$ tests; $p<0.0001$ ). In pb2, response to Nonanoic acid is significantly higher than to $1-$ Nonanol ( $n=10$ palps; ANOVA with post hoc $t$ tests; $p=0.0110)$. Data was mean with SEM. C Quantification of mean responses to dose series of E-2-Hexenal and Hexanal in pbl ( \pm SEM; $n=12$ palps). D Quantification of mean responses to dose series of E-2-Hexenal and Hexanal in pb2 $( \pm S E M ; n=10$ palps) 


\section{Identification of IR8a and obp2a required for detection of Hexanal}

Dendritic localization of specific ORs requires Orco, and mutation of Orco removes behavioral responses toward many odorants [32]. However, in our case Orco RNAi failed to abolish responses for two aldehydes completely, implying more than one pathway may mediate aldehydes. It's interesting to investigate IRs-involved olfactory circuits on palps. Firstly, RT-PCR presented that IR8a was highly expressed across both antennae and palp with similar abundance (Figure 5A, top) and its cellular localization was restricted in arc-like, abundant neuron populations, resembling dominant sensory neurons innervated by chaetica (Figure 5A, bottom left). This spatial distribution was in contrast with limited pattern of OR2 (Figure 3A, B). Immunohistochemistry showed that antennal chaetica lymph was strongly labeled by antibody against OBP2a [21]. RT-PCR and hybridization provided more evidences that obp2a was transcribed equivalently between two organs and large number of cavity-like structure were marked in close proximity to the base of chaetica (Figure 5A, top and bottom right). In comparison with obp1, obp2a occupied wide and differentiated cell populations in chaetica, instead of basiconica. Furthermore, co-localization staining supported that cells expressing IR8a and obp2a were clustered in one sensilum (Figure 5B), similar to OR2 and obp1 depicted in Figure 3A.

Then we treated locusts with dsRNA directing either IR8a or obp2a and subsequent POR experiments revealed only index of Hexanal, rather than the other 6 compounds was significantly attenuated after regulating either IR8a or obp2a (Figure 5C, D). Dual dsRNAs were injected simultaneously and this triggered mild but not significantly worse POR impairment than single IR8a RNAi for Hexanal (Figure 5E), though no unspecific interference was observed (Figure 5G). This suggests that IR8a and obp2a function in tandem, not parallel to recognize Hexanal in palp chaetica. Previously, it's demonstrated that Hexanal is detected via OR2-obp1 in basiconica and we speculated that Hexanal could be processed in combinatorial way by both IR and OR. Animals with dual dsRNAs directing IR8a and Orco were examined in POR and the minimum index to Hexanal was observed, which was significantly lower than single treatment of either co-receptors (Figure $5 F)$. However, dual injections failed to abolish the activity to E-2-Hexenal any further, and were not significantly different to Orco-RNAi. Consistent with Figure 5C, impairing IR8a maintained the high POR response to E-2-Hexenal, highlighting that two olfactory pathways contribute to distinguish saturated and unsaturated 6 carbon aldehydes on palps: IR8a mediated only saturated Hexanal, while Orco-mediated olfactory circuitry tuned responses to both. None of the other compounds, including ester, alcohol and ketone, presented distinguishable POR defects compared with wild type animals. Furthermore, no unspecific interference against either Orco or OR2 was observed after IR8a injections (Figure 5H). Taken together, our molecular and behavioral results are consistent with a model in which olfactory detections to aldehydes on locust palps rely on two independent but partially integrated tuning pathways.

\section{Discussion}

\section{Quantitative measurement of mouthpart olfactory responses}

In this paper, we described a behavioral paradigm (POR) to sensitively and specifically quantify olfactory responses on the locust mouthparts, which was used to investigate memory acquisition in locust before [27]. While the mouthparts are widely argued for taste reception, we presented olfactory roles. A recent study in the malaria vector, Anopheles gambiae demonstrated that limited human-related scents are detected via a small set of labial olfactory sensilla, supporting the hypothesis that the mosquito labellum confers potential olfactory function [33]. However, the conclusion is drawn from electrophysiological recordings only, and the molecular basis and behavioral significance remain unknown. In Drosophila, proboscis extension reflex (PER) is widely used to measure the aversive or attractive characters of a taste stimulus. Wang, et al [34] exploited this assay and demonstrated that Gr5a and Gr66a are responsible for appetitive response to sugar and aversive response to bitter respectively. Here, we provided a quantitative assay to measure the olfactory discrimination between behaviorally active and inert scents on the palps, the commonly recognized taste organ. This setup allows to depict olfactory recognition on palps, analogous to a biological decoder. Extracellular recording results, which Hexanal and E-2-Hexenal elicited neuronal activities in palp basiconica (Figure 4), also supported that active compounds identified from POR were directly activate olfactory pathways on the palps. In addition, certain compounds remain active behaviorally after knocking down LmigOrco and LmigIR8a (Figure 3 and 5) and at least two explanations could be assumed. First, RNA interference cannot remove all transcripts of target genes completely and that means the residual 
co-receptor could continue signal trafficking on the membrane to certain compounds, if not all. The expression thresholds of co-receptor required for normal olfactory recognition could also be differential to different compounds. Second, indeed, more than one chemosensory receptor families are identified on the palps based on transcriptome sequencing (not published), including 19 candidate Pickpocks genes, 5 candidate Gustatory Receptor genes (concerning low expression level similar to Drosophila melanogaster, hard to acquire the full or large part of the GRs repertoire [35]) and 20 Ionotropic Receptor genes, including LmigIR25a and LmigIR93a. It's possible that other chemosensory receptor families, like LmigIR25a and LmigGRs contribute to the molecular coding of the remaining compounds, rather than LmigIR8a and LmigOrco. Besides, we do not know valences or the ecological significances (attraction or aversion) governed by those active compounds, though our results suggest an importance to the animal. Detailed tracking of tip movement pattern could facilitate to understand what they mean for animals. Several parameters related with POR, like opening frequency, duration and latency, as well as distance between paired tips are proved to be of significance in investigating behavioral correlates of neural synchrony [36].

\section{Olfactory responses in gustatory organs}

This study confirmed and extended the conclusion that odorant receptors are expressed and function in olfactory process on the conventional gustatory organs in Locusta migratoria, though, unexpectedly. An easy-to-use paradigm, POR, similar to PER for gustatory evaluation in Drosophila, screened variety of active compounds according to palp opening in a way of airborne stimulation. RNAi efficiently knocked down the expression of chemosensory molecules and subsequently behavioral dysfunction was observed. Moreover, SSR response to aldehydes in palp basiconica showed that only a small specific sensilla subtype presented significant electrophysiological activity, rather than all others. Taken together, these results strongly suggest that palp olfactory ORNs are bona fide responsible for volatiles detection.

Morphologically, chemosensory sensilla for odorant and taste are apparently distinguishable. For example, taste sensilla are classified to be those which are thick-walled with an apical pore and relatively few dendrites, whereas the olfactory sensilla are those with a thin cuticle, many pores and a multitude of dendrites [37, 38]. However, behavioral observations from several reports [39-41] indicate such classification based on morphology cannot always apply. For example, tobacco hornworm of Manduca sexta employs lateral sensilla styloconica on the maxillae to respond to natural vapors from an average distance of $100 \mu \mathrm{m}$ and this capacity is sensilla specific to lateral styloconica, rather than medial ones. However, tip recordings suggest that those lateral styloconica respond to sucrose and salts sensitively which demonstrate they are gustatory sensilla [42]. This result is consistent with the notion that gustatory senilla possess molecular elements for olfactory sensation. Considering the morphological contrast, Hiroi et al ectopically expressed one OR complex in labial gustatory receptor neuron (GRNs) via GAL4/UAS expression system and those genetically modified GRNs can sense cognate odors of OR at close range in the aerial phase [43]. That means ORs are functional in taste neurons and morphological structure of taste sensilla is capable of allowing odor's entrance into sensill lymph to elicit neuronal excitements. Nevertheless, it is technically challenging to record electrically on palp chaetica in locust partially caused by thick wall and socket in the sensilla base. Since large IRs repertoires, including antennal variant IRs are identified on palp chaetica and RNAi against IR8a significantly induce behavioral defects to vapor stimulation, it is likely to imply the contribution of chaetica to olfactory signaling. This olfactory capacity of gustatory sensilla allow insects to continuously monitor the food chemistry in close range even though they might not actually contact, as the case reported in tobacco hornworm of Manduca sexta [42].

\section{One odor tuned with distinct receptor families}

POR data demonstrates that Hexanal are tuned by two distinct receptor pathways on the locust mouthparts; one is mediated by OR-expressing neurons in basiconica, and another is by IR-positive neurons in chaetica. In contrast, E-2-Hexenal are detected exclusively by basiconica ORNs. From an evolutionary view, IRs is much older than ORs, with the latter emerging after the appearance of winged insects [44]. Antennal IRs, a subfamily of the divergent IRs, are likely to be the first olfactory receptors in insects, and they served as odorant receptors with broad scent tunings in the ancestral linages of insects [6]. However, while ORs and IRs mostly detect distinct compounds, there are certain overlapping responses in insects. One quantitative investigation of ligand preferences toward sensilla types via either summed response profile or distribution in physicochemical space showed clear tunings distinction for very most tested odors; while few of compounds, including 2,3-butanedione and phenylacetaldehyde are encoded by both IRs and ORs 
in similar spiking activity [45]. 2,3-butanedione is tuned by two distinct receptor families with equivalent spiking intensity, e.g., IR75a, IR64a, OR42a and OR92a according to DoOR [46], whereas, the functional combinations remain unclear. As an important host plant GLV [47], Hexanal possibly contribute to further confirmation to food source by oligophages locust in parallel pathways in order to

A

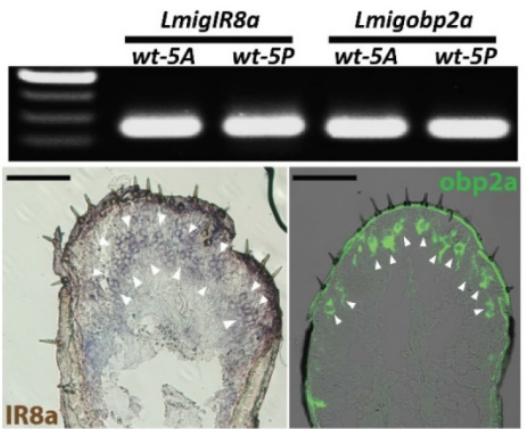

C

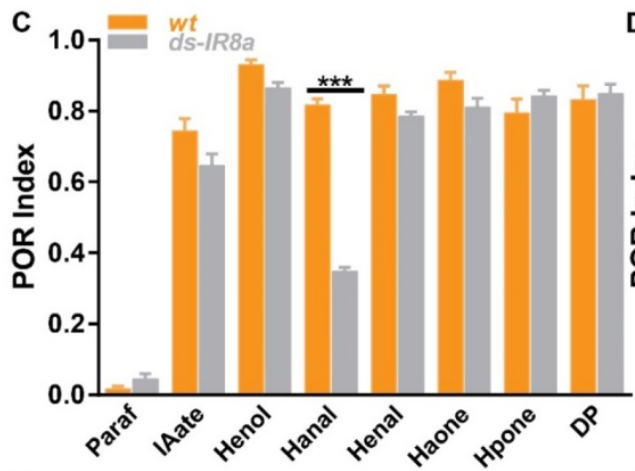

B enhance the specificity and sensitivity. Because of lacking detailed molecular information for all IRs on the palps, we're not allowed to investigate the specific IR mediating this aldehyde. In vivo two-color localization between IR8a and unknown IRs could provide more hints for further functional consolidation.

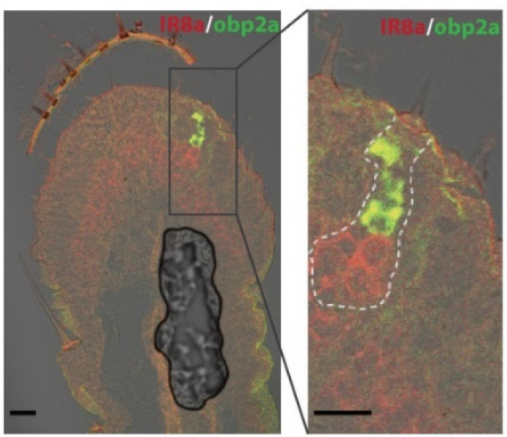

D

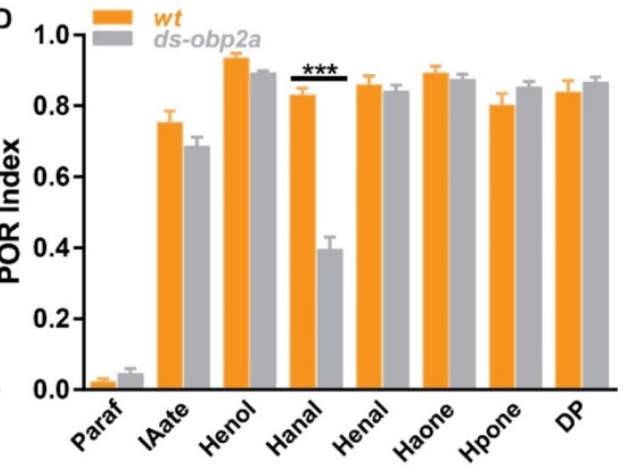

$\mathbf{F}$

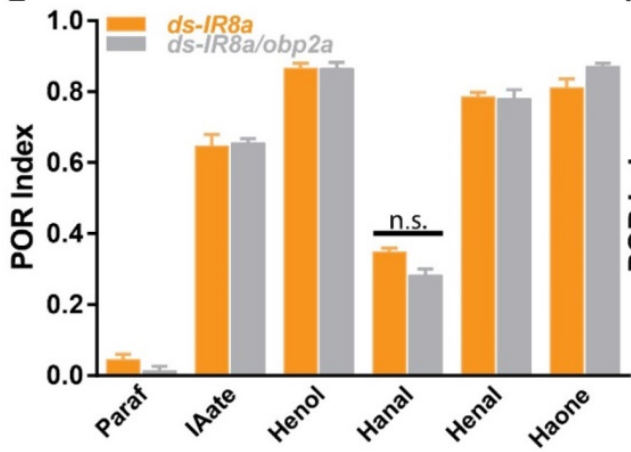

F 1 -Orco
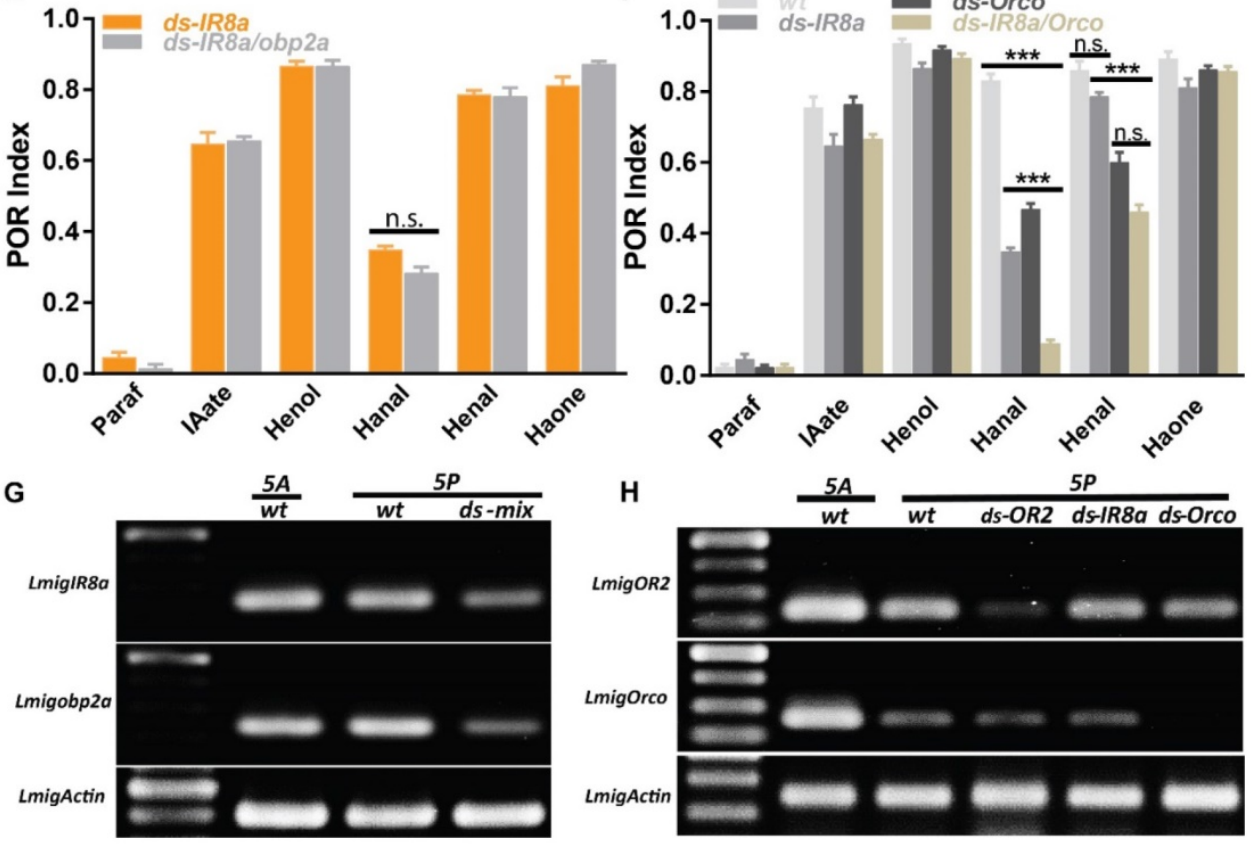

Figure 5. IR8a and OR2 pathways cooperatively regulate aldehyde perception. A IR8a and obp2a are expressed abundantly on the palp. Top, RT-PCR with wildtype $5^{\text {th }}$ instar antennal and palp cDNA; Bottom, single color in situ hybridization on the $5^{\text {th }}$ instar palp cryosection. Arrowhead indicates positive cell clusters for each gene. Bar, $100 \mu \mathrm{m}$. B Double color in situ hybridization between IR8a and obp2a on the $5^{\text {th }}$ instar palp cryosection. Left, merged staining on the palp; Right, enlarged view from left frame area, dashed line indicating potential cell clusters from one sensilla. Bar, $25 \mu \mathrm{m}$. C POR assays toward multiple compounds with locusts treated with water or ds RNA of IR8a. N=8-9 (30 locusts each replicate) for each assay. D POR assays toward multiple compounds with locusts treated with water or ds RNA of obp2a. $\mathrm{N}=9$ (30 locusts each replicate) for each assay. E POR assays toward multiple compounds with locusts treated with ds RNA mix of IR8a and obp2a. N=6-9 (30 locusts each replicate) for each assay. F POR assays toward multiple compounds with locusts treated with individual ds RNA or mix of IR8a and Orco. $N=8-9$ (30 locusts each replicate) for each assay. *** $p<0.001$, two-way ANOVA with Bonferroni post-tests. G Semi-quantitative RT-PCR to confirm RNAi silencing efficiency and specificity for ds RNA mix of IR8a and obp2a. H Similar as G, no cross-silencing between Orco and IR8a pathways with semi-quantitative RT-PCR. Actin was used to check template quality. Paraf: Paraffin oil; IAate: iso-amyl acetate; Henol: Z-3-Hexenol; Hanal: Hexanal; Henal: E-2-Hexenal; Haone: 2-Hexanone; Hpone: 2-Heptanone; DP: 2,5-Dimethylpyrazine 


\section{Abbreviations}

OR: Odorant receptor; IR: Ionotropic receptor; OBP: Odorant binding protein; ORN: Olfactory receptor neuron; GRN: Gustatory receptor neuron; POR: Palp opening response; GLV: Green leaf volatiles; iGluRs: Ionotropic glutamate receptors; RNAi: RNA interference; FISH: Fluorescence in situ hybridization.

\section{Supplementary Material}

Additional File 1:

Supplementary figures and tables.

http://www.ijbs.com/v13p0759s1.pdf

Additional File 2:

Video S1. http://www.ijbs.com/v13p0759s2.avi

Additional File 3:

Video S2. http://www.ijbs.com/v13p0759s3.avi

Additional File 4:

Video S3. http://www.ijbs.com/v13p0759s4.avi

Additional File 5:

Video S4. http://www.ijbs.com/v13p0759s5.avi

\section{Acknowledgements}

We thank Bo Gao, Shuang Shan and Xiao Xu for technical assistance with in situ hybridization; we also give thanks to Dr. Ewald Grosse-Wilde and Prof. Dr. Bill S. Hansson for critical comments and language help. This work is supported by the grants from National Natural Science Foundation of China (No. 31472037) and the China Scholarship Council.

\section{Author contributions}

Conceived the idea: LW.Z, L.Z. Experimental design and data acquisition: LW.Z, HW.L. Data analysis and interpretation: LW.Z, HW.L, L.Z. First draft of manuscript: LW.Z, L.Z. Comments and revision: LW.Z, HW.L and L.Z.

\section{Competing Interests}

The authors have declared that no competing interest exists.

\section{References}

1. Larsson MC, Domingos AI, Jones WD, Chiappe ME, Amrein H, Vosshall LB. Or83b encodes a broadly expressed odorant receptor essential for Drosophila olfaction. Neuron. 2004; 43: 703-14.

2. Vosshall LB, Amrein H, Morozov PS, Rzhetsky A, Axel R. A spatial map of olfactory receptor expression in the Drosophila antenna. Cell. 1999; 96: 725-36.

3. Benton R, Vannice KS, Gomez-Diaz C, Vosshall LB. Variant ionotropic glutamate receptors as chemosensory receptors in Drosophila. Cell. 2009; 136: 149-62.

4. Leal WS. Odorant reception in insects: roles of receptors, binding proteins, and degrading enzymes. Annual review of entomology. 2013; 58: 373-91.

5. Benton R, Sachse S, Michnick SW, Vosshall LB. Atypical membrane topology and heteromeric function of Drosophila odorant receptors in vivo. PLoS biology. 2006; 4: 240-57.

6. Missbach C, Dweck HK, Vogel H, Vilcinskas A, Stensmyr MC, Hansson BS, et al. Evolution of insect olfactory receptors. Elife. 2014; 3: e02115.
7. Wicher D, Schafer R, Bauernfeind R, Stensmyr MC, Heller R, Heinemann SH, et al. Drosophila odorant receptors are both ligand-gated and cyclic-nucleotide-activated cation channels. Nature. 2008; 452: 1007-11.

8. Sato K, Pellegrino M, Nakagawa T, Nakagawa T, Vosshall LB, Touhara K. Insect olfactory receptors are heteromeric ligand-gated ion channels. Nature. 2008; 452: 1002-6.

9. Croset V, Rytz R, Cummins SF, Budd A, Brawand D, Kaessmann H, et al. Ancient protostome origin of chemosensory ionotropic glutamate receptors and the evolution of insect taste and olfaction. PLoS genetics. 2010; 6: e1001064.

10. Abuin L, Bargeton B, Ulbrich MH, Isacoff EY, Kellenberger S, Benton R. Functional architecture of olfactory ionotropic glutamate receptors. Neuron. 2011; 69: 44-60.

11. Yao CA, Ignell R, Carlson JR. Chemosensory coding by neurons in the coeloconic sensilla of the Drosophila antenna. The Journal of neuroscience. 2005; 25: 8359-67.

12. Liu C, Pitts RJ, Bohbot JD, Jones PL, Wang GR, Zwiebel LJ. Distinct olfactory signaling mechanisms in the malaria vector mosquito Anopheles gambiae. PLoS biology. 2010; 8: 697-704.

13. Hansson BS, Stensmyr MC. Evolution of insect olfaction. Neuron. 2011; 72: 698-711.

14. Chapman RF. Contact chemoreception in feeding by phytophagous insects. Annual review of entomology. 2003; 48: 455-84.

15. Chapman R, Sword G. The importance of palpation in food selection by a polyphagous grasshopper (Orthoptera: Acrididae). Journal of insect behavior. 1993; 6: 79-91.

16. Winstanley $\mathrm{C}$, Blaney W. Chemosensory mechanisms of locusts in relation to feeding. Entomologia Experimentalis et Applicata. 1978; 24: 750-8.

17. Blaney W, Duckett A. The significance of palpation by the maxillary palps of Locusta migratoria (L): an electrophysiological and behavioural study. The Journal of experimental biology. 1975; 63: 701-12.

18. Blaney W. Electrophysiological responses of the terminal sensilla on the maxillary palps of Locusta migratoria (L.) to some electrolytes and non-electrolytes. Journal of experimental Biology. 1974; 60: 275-93.

19. Jin X, Zhang SG, Zhang L. Expression of odorant-binding and chemosensory proteins and spatial map of chemosensilla on labial palps of Locusta migratoria (Orthoptera: Acrididae). Arthropod Struct Dev. 2006; 35: 47-56.

20. Xu H, Guo M, Yang Y, You Y, Zhang L. Differential expression of two novel odorant receptors in the locust (Locusta migratoria). BMC neuroscience. 2013; 14: 50 .

21. Yu F, Zhang S, Zhang L, Pelosi P. Intriguing similarities between two novel odorant-binding proteins of locusts. Biochem Biophys Res Commun. 2009; 385: 369-74.

22. Ban L, Scaloni A, D'ambrosio C, Zhang L, Yan Y, Pelosi P. Biochemical characterization and bacterial expression of an odorant-binding protein from Locusta migratoria. Cellular and molecular life sciences. 2003; 60: 390-400.

23. Dweck HKM, Ebrahim SAM, Khallaf MA, Koenig C, Farhan A, Stieber R, et al. Olfactory channels associated with the Drosophila maxillary palp mediate short- and long-range attraction. eLife. 2016; 5: e14925.

24. Luo Y, Wang X, Wang X, Yu D, Chen B, Kang L. Differential responses of migratory locusts to systemic RNA interference via double-stranded RNA injection and feeding. Insect molecular biology. 2013; 22: 574-83.

25. Roessingh P, Simpson SJ, James S. Analysis of phase-related changes in behaviour of desert locust nymphs. Proceedings of the Royal Society of London Series B: Biological Sciences. 1993; 252: 43-9.

26. Blaney WM, Chapman RF. The function of the maxillary palps of Acrididae (Orthoptera). Entomologia Experimentalis et Applicata. 1970; 13: 363-76.

27. Simoes P, Ott SR, Niven JE. Associative olfactory learning in the desert locust, Schistocerca gregaria. The Journal of experimental biology. 2011; 214: 2495-503.

28. Li J, Zhang L, Wang X. An odorant-binding protein involved in perception of host plant odorant in locust Locusta migratoria. Arch Insect Biochem Physiol. 2016; 91: 221-9.

29. Guo M, Krieger J, Wilde E-G, Mißbach C, Zhang L, Breer H. Variant ionotropic receptors are expressed in olfactory sensory neurons of coeloconic sensilla on the antenna of the desert locust (Schistocerca gregaria). International Journal of Biological Sciences. 2014; 10: 1-14.

30. Wang Z, Yang P, Chen D, Jiang F, Li Y, Wang X, et al. Identification and functional analysis of olfactory receptor family reveal unusual characteristics of the olfactory system in the migratory locust. Cellular and molecular life sciences : CMLS. 2015; 72: 4429-43.

31. Yang Y, Krieger J, Zhang L, Breer H. The olfactory co-receptor Orco from the migratory locust (Locusta migratoria) and the desert locust (Schistocerca gregaria): identification and expression pattern. International journal of biological sciences. 2012; 8: 159-70.

32. Fishilevich E, Domingos AI, Asahina K, Naef F, Vosshall LB, Louis M. Chemotaxis behavior mediated by single larval olfactory neurons in Drosophila. Current biology. 2005; 15: 2086-96.

33. Kwon HW, Lu T, Rutzler M, Zwiebel LJ. Olfactory responses in a gustatory organ of the malaria vector mosquito Anopheles gambiae. Proceedings of the National Academy of Sciences of the United States of America. 2006; 103: 13526-31.

34. Wang Z, Singhvi A, Kong P, Scott K. Taste Representations in the Drosophila Brain. Cell. 2004; 117: 981-91.

35. Clyne PJ. Candidate Taste Receptors in Drosophila. Science. 2000; 287: 1830-4. 
36. Saha D, Li C, Peterson S, Padovano W, Katta N, Raman B. Behavioural correlates of combinatorial versus temporal features of odour codes. Nature Communications. 2015; 6: 6953.

37. Slifer EH. The Structure of Arthropod Chemoreceptors. Annual review of entomology. 2003; 15: 121-42.

38. Stocker RF. The organization of the chemosensory system in Drosophila melanogaster: a review. Cell and Tissue Research. 1994; 275: 3-26.

39. Hanson FE, Dethier VG. Role of gustation and olfaction in food plant discrimination in the tobacco hornworm, Manduca sexta. J Insect Physiol. 1973; 19: 1019-34.

40. Zacharuk RY, Mittler TE, Radovsky FJ, Resh VH. Ultrastructure and function of insect chemosensilla. Annual review of entomology. 2003; 25: 27-47.

41. Dethier VG. Sensitivity of the contact chemoreceptors of the blowfly to vapors. Proceedings of the National Academy of Sciences of the United States of America. 1972; 69: 2189-92.

42. Städler E, Hanson FE. Olfactory capabilities of the "gustatory" chemoreceptors of the tobacco hornworm larvae. Journal of comparative physiology. 1975; 104: 97-102.

43. Hiroi M, Tanimura T, Marion-Poll F. Hedonic taste in Drosophila revealed by olfactory receptors expressed in taste neurons. PloS one. 2008; 3: e2610.

44. Croset V, Rytz R, Cummins SF, Budd A, Brawand D, Kaessmann H, et al. Ancient protostome origin of chemosensory ionotropic glutamate receptors and the evolution of insect taste and olfaction. PLoS genetics. 2010; 6: e1001064.

45. Silbering AF, Rytz R, Grosjean Y, Abuin L, Ramdya P, Jefferis GS, et al. Complementary function and integrated wiring of the evolutionarily distinct Drosophila olfactory subsystems. The Journal of neuroscience : the official journal of the Society for Neuroscience. 2011; 31: 13357-75.

46. Münch D, Galizia CG. DoOR 2.0 - Comprehensive mapping of Drosophila melanogaster odorant responses. Scientific reports. 2016; 6: 21841

47. Buttery RG, Ling LC. Corn leaf volatiles: Indentification using Tenax trapping for possible insect attractants. Journal of Agricultural and Food Chemistry. 1984; 32: 1104-6. 